\title{
Rethinking the use of finite element simulations in comparative biomechanics research
}

\author{
Z. Jack Tseng ${ }^{\text {Corresp. } 1}$ \\ ${ }^{1}$ Department of Integrative Biology and Museum of Paleontology, University of California, Berkeley, Berkeley, CA, United States \\ Corresponding Author: Z. Jack Tseng \\ Email address: zjt@berkeley.edu
}

In the past 15 years, the finite element (FE) method has become a ubiquitous tool in the repertoire of evolutionary biologists. The method is used to estimate and compare biomechanical performance implicated as selective factors in the evolution of morphological structures. A feature common to many comparative studies using 3D FE simulations is small taxonomic sample sizes. The time-consuming nature of FE model construction is considered a main limiting factor in taxonomic breadth of comparative FE analyses. Using a composite FE model dataset, I show that the combination of small taxonomic sample sizes and comparative FE data in analyses of evolutionary associations of biomechanical performance to feeding ecology generates artificially elevated correlations. Such biases introduce false positives into interpretations of clade-level trends. Considering this potential pitfall, recommendations are provided to consider the ways FE analyses are best used to address both taxon-specific and clade-level evolutionary questions. 
1 Rethinking the use of finite element simulations in comparative biomechanics research

3 Z. Jack Tseng

4

5 Department of Integrative Biology and Museum of Paleontology

6 University of California, Berkeley

7 zjt@berkeley.edu

8 


\section{Abstract}

In the past 15 years, the finite element (FE) method has become a ubiquitous tool in the repertoire of evolutionary biologists. The method is used to estimate and compare biomechanical performance implicated as selective factors in the evolution of morphological structures. A

13 feature common to many comparative studies using 3D FE simulations is small taxonomic

14 sample sizes. The time-consuming nature of FE model construction is considered a main limiting

15 factor in taxonomic breadth of comparative FE analyses. Using a composite FE model dataset, I

16 show that the combination of small taxonomic sample sizes and comparative FE data in analyses

17 of evolutionary associations of biomechanical performance to feeding ecology generates

18 artificially elevated correlations. Such biases introduce false positives into interpretations of

19 clade-level trends. Considering this potential pitfall, recommendations are provided to consider

20 the ways FE analyses are best used to address both taxon-specific and clade-level evolutionary

21 questions. 
24

25

26

27

\section{Introduction}

Structure-function relationships underlie many hypotheses about patterns of morphological disparity in organisms (Lauder 1981; Lauder and Thomason 1995). Among the major tools in estimating the functional performance of vertebrate structures in particular is the use of biomechanical simulations to predict traits like bite force, structural stiffness, mechanical efficiency, etc. (Richmond et al. 2005; Ross 2005). In the past 15 years, the adaptation of finite element (FE) modeling, an engineering method for solving load-deformation scenarios using principles of continuum mechanics, to biological questions has been greatly expanded across the broad spectrum of organismal study systems. An important feature of FE approaches is the ability to quantitatively test functional morphological hypotheses, contrasting it with largely qualitative conclusions and inferences in classic comparative and functional anatomical approaches A search in the 'Web of Science' database returned 768 publications between 2005 and 2020 on using FE models in the study of evolution and biology (Fig 1A). Research using FE analysis of the vertebrate skeleton covers topics such as inferring locomotory and masticatory performance in the vertebrate fossil record (Rayfield 2007), morphofunctional evolution in entire clades (Pierce et al. 2008), to evolutionary optimization of functional morphological attributes (Polly et al. 2016), and others.

Studies using the FE method to test hypotheses about vertebrate structure-function fall into two major categories: 2D and 3D analyses. 2D FE models are typically derived from photographs of specimens, whereas 3D FE models are typically derived from computed tomography $(\mathrm{CT})$ or surface scans. The main trade-off between 2D and 3D approaches is model sample size versus time investment in building each model (Morales-García et al. 2019). 2D models are quicker to build and allow for larger taxonomic sample sizes, but the extent of model 
47 simplification restricts its application to structures whose function can be reasonably

48

49 approximated in two dimensions. 3D models can provide a fuller characterization of the morphology at hand but are much more time-consuming to build. As such, most studies using 3D FE models examine fewer than 10 taxa (Fig. 1B).

Given the steady increase in research studies employing FE methods to address comparative biomechanical and evolutionary questions, it is critical for both practitioners of FE analyses and researchers considering using the FE toolkit to test biomechanical hypotheses to be able to design studies efficiently using this time-intensive method. To highlight the important issue of sample size in 3D FE studies of structure-function relationships, here I ask the question: are time-bottlenecked small-sample 3D FE datasets adequate in reproducing performanceecology correlations observed in broader taxonomic datasets? Given the already rich literature in using 3D FE analyses in comparative skull biomechanics research, I take a meta-analysis approach to addressing this question using mainly published studies.

The study system I use to demonstrate the effect of taxonomic sample size on performance-ecology relationships is the skull. Although the FE method is applicable to any morphological system whose geometry and biophysical boundary conditions can be digitized and parameterized, most studies in vertebrates employing FE modeling in a comparative context have done so to study skull biomechanical performance (Ross 2005). Nevertheless, the effects of taxonomic sample size are expected to be shared in part by other study systems. Therefore, the findings from these analyses are expected to be relevant to researchers in comparative biology, paleobiology, bioengineering, and biomedical engineering fields that use multi-taxon comparative FE datasets to test structure-function hypotheses. 
70

71

72

73

74

\section{Survey Methodology}

I tallied the number of taxa included in published FE studies in general (Fig. 1A) and specifically of the vertebrate skull from 2005 to 2020 for 3D-model based analyses (Fig. 1B). Total number of peer-reviewed publications using FE methods were extracted from the Web of Science database (accessed 22 December 2020) using the key words "finite element" + "evolution" + bio*.

A second survey of the literature was conducted on FE studies that specifically address vertebrate skull biomechanics in a comparative context by searches in both Web of Science and Google Scholar (both accessed 15 December 2020) using key words "finite element" + "skull” + phylo*. The year of publication was constrained to between 2005 and 2020 . The total number of unique species studied in each surveyed publication was counted. The surveyed publications were further vetted by removing all studies that used 2D FE models (Fig. 1B). Out of the 28 studies obtained from the skull FE survey (Table S1), the FE model construction methodology used in each survey publication was noted and a composite FE model dataset was constructed (see next section).

\section{Meta-analysis Methodology}

Based on the surveyed publication dataset, I compiled model results from Prybyla et al. (2018), Perez-Ramos et al. (2020), as well as three additional, new models that complement those in the two published studies to assemble a dataset of 3D cranial FE output data (here on referred to as the 'full dataset'; Table 1). Data from those studies were chosen because together they represent the largest sample of FE models in the literature constructed using a single 
92 protocol, thus reducing confounding factors from differences in FE model construction

93 methodology and software programs used. The full dataset included published data from FE

94 models of 24 carnivoran species and new data from 3 species (Aonyx capensis, Hydrictis

95 maculicollis, and Lutra lutra). The new models were built following Perez Ramos et al.'s (2020)

96 protocol and summarized below. Sixteen extant species FE models out of 21 taxa from Prybyla

97 et al. (2018) were included; five taxa from that study were excluded (Ursus arctos and $U$.

98 maritimus overlapped with the Perez Ramos et al. study, and Leptarctus primus, Thinocyon

99 velox, and Oodectes herpestoides were excluded because they represent fossil taxa without

100 ecological trait data). Likewise, 4 of 12 taxa from Perez Ramos et al. (2020) were excluded

101 because they represented fossil taxa (U. ingressus, U. spelaeus spelaeus, $U$. spelaeus eremus, $U$.

102 spelaeus ladinicus).

The FE model building protocol was identical across all 27 models used in this study except for the elastic moduli values (20 GPa for models from Prybyla et al., 2018, $18 \mathrm{GPa}$ for models from Perez Ramos et al., 2020 and this study), which were standardized using a secondary linear regression analysis. Briefly, the FE model and simulation protocol include capturing the 3D geometry of each skull specimen using CT scanning (data for the new models constructed were downloaded from scans uploaded to MorphoSource.org by Tseng et al. 2017).

Three-dimensional skull models were constructed from voxels selected using threshold segmentation to include all cortical bone in Avizo (Thermo Fisher Scientific, Hillsboro, Oregon, 
115 meshes were constructed using 4-noded tetrahedral elements in Strand7 (Strand7 Pty. Ltd,

116 Sydney, Australia). Input muscle forces were simulated using muscle forces estimates derived

117 from a modified dry skull method (Thomason 1991) for estimating muscle attachment areas of

118 the temporalis, masseter, and medial pterygoid muscles. Force vectors mimicking muscle

119 wrapping over the insertion sites were generated using the BoneLoad MatLab script (Grosse et

120 al. 2007).

New models generated in this study used elastic moduli of $18 \mathrm{GPa}$ as in Perez Ramos et al. (2020); models from Prybyla et al. (2018) used the slightly higher $20 \mathrm{GPa}$ as their elastic moduli. As such, moduli correction was necessary to directly compare the outputs of the simulations from the different studies. I sampled 9 taxa from the Prybyla et al. (2018) dataset and reanalyzed the models using elastic moduli of $18 \mathrm{GPa}$. The resulting deviations were then correlated using linear regression analyses to obtain correction factors for $20 \mathrm{GPa}$-based data to their $18 \mathrm{GPa}$ equivalent values (Figure S1; Table S2). The resulting relationships between 18 $\mathrm{GPa}$ and $20 \mathrm{GPa}$ data are adequately described by linear relationships $\left(\mathrm{R}^{2}=0.99\right.$ for all output values sampled, see next paragraph). muscle force (including temporalis, masseter, and medial pterygoid muscle forces, in Newtons), mechanical efficiency (bite nodal restraint reaction force/total muscle input force) at the canine and fourth premolar teeth, respectively, and overall skull strain energy (a measure of work done to deform the skull during simulated bites, in Joules) in canine bite and fourth premolar bite scenarios, respectively (Table 1). All FE simulations portrayed unilateral bites using homogeneous and isotropic material property models, solved by linear static analysis. For a more detailed explanation of the FE modeling workflow see reviews by Ross (2005) and Rayfield 
138 (2007). For carnivorans and mammals in general, bite force (the magnitude of reaction forces 139 that can be generated at the tooth-food interface, often measured in Newtons) and the related 140 measure of mechanical efficiency (ME; the relative amount of bite force generated per unit of 141 input muscle force, a unit-less ratio) are important biomechanical traits that broadly correlate 142 with feeding ecology (Wroe et al. 2005). Strain energy (SE), the amount of work done in 143 deforming a structure (as in deformation of the skull during biting), is thought to be a variable 144 that represents how 'energy efficient' a biological structure is in converting input force towards 145 output force rather than towards deforming itself (Dumont et al. 2009). The combination of these 146 two measures of biomechanical performance was previously found to correlate with omnivory 147 (gradual decrease in SE with increasing ME from the anterior to the posterior toothrow) and 148 dietary specialization (presence of noticeable drops in SE in specialized tooth positions as ME 149 increases)(Tseng and Flynn 2015). These observations were subsequently summarized by Perez 150 Ramos et al. (2020) as relatively SE-invariant increase of ME from anterior to posterior dentition 151 in generalists versus the SE-varying changes in ME for dietary specialists adapted to using 152 specific tooth loci for feeding tasks. For the reasons outlined above, in this study I focus on ME and SE values of the canine and fourth premolar tooth loci as biomechanical traits that are expected to correlate with dietary breadth (range of food items and therefore food mechanical properties consumed) and trophic level (herbivore and carnivores being more specialized than omnivores). In the extracted dataset,

157 strain energy values were corrected for model volume and input muscle force area differences according to the equation provided by Dumont et al. (2009). The first model in the alphabetically

159 arranged dataset, Ailuropoda melanoleuca, was arbitrarily chosen as the standard model to which 160 all other model strain energy values are adjusted to. The final set of biomechanical 
161 characteristics calculated for all 27 taxa in the full dataset included ACME (Adjusted Canine

162 mechanical efficiency), AP4ME (Adjusted fourth premolar mechanical efficiency), ADJCSE

163 (adjusted strain energy value in canine bite scenario), and ADJP4SE (Adjusted strain energy

164 value in fourth premolar bite scenario). These data served as the functional performance

165 variables used to characterize a given taxon (Table 1).

The feeding ecological variables used for correlation to the performance variables include

167

168

169

170

171

172

173

174

175

176

177

178

179

180

181

182

183 dietary breadth and trophic level. The definition of the levels in each variable and the coding for each of the taxon included in the dataset are taken from the PanTHERIA database (Jones et al. 2009) (Table 2). Feeding ecological grouping in this study is characterized by the combination of these two categories.

To formalize the qualitative association between performance variables and feeding ecological categories employed in many published studies, I used hierarchical clustering to group the taxa. One dendrogram each was calculated for the FE outputs (performance variables) and dietary ecological traits (dietary breadth and trophic level). The former, continuous multivariate dataset was clustered using Ward's distance measure on an Euclidean distance matrix calculated from the four FE output variables. The latter, categorical bivariate dataset was clustered also using Ward's distance measure, but on a Gower's distance matrix for discrete variables (Gower 1971).

A non-parametric Baker's Gamma correlation coefficient (Baker 1974) was calculated from the two resulting cluster dendrograms to establish the degree of association between the performance variable groupings and dietary ecology groupings of the full dataset. Baker's Gamma counts the level (designated by $\mathrm{k}$, the number of clusters) on the dendrogram at which a given pair of taxa is grouped together in both dendrograms, followed by a Spearman (non-

Peer) reviewing PDF | (2020:12:56771:1:0:NEW 16 Feb 2021) 
184 parametric) correlation coefficient calculation. This index relies only on the topology of the 185 dendrograms, but not their distance or branch lengths. To assess the influence of branch length

186 information on the resulting correlation measurement, the analysis was repeated using the

187 cophenetic correlation coefficient instead of Baker's Gamma (Sokal and Rohlf 1962). The 95\%

188 confidence interval around the calculated correlation coefficient was estimated using bootstrap

189 resampling by simulating a sample of 1,000 dendrograms each in the performance and dietary

190 ecology datasets with randomly assigned tip names from the shared 27-taxon name list. Baker's

191 Gamma and cophenetic correlation coefficients were calculated for each pair of the 1,000

192 simulated performance and ecology dendrograms. A 95\% confidence interval was then

193 calculated from those correlation coefficients.

Next, a series of correlation coefficients between performance and ecology dendrograms

were calculated in incrementally smaller sub-datasets of the full dataset of 27 taxa. 1,000

bootstrap samples were generated for each of 24 sets of bootstrap samples, from 26 taxa (1 fewer

taxon than full dataset) to 3 taxa (minimum to polarize pairwise comparisons) per dataset. The

bootstrapped datasets were pulled from the full dataset with replacement. Median, quantile

ranges, mean, and 95\% confidence intervals of the mean were calculated for bootstrap replicates level. 
207 respectively, the functional and ecological cluster association to phylogenetic grouping was also

208 assessed within the same analytical framework. A phylogeny with branch lengths based on

209 molecular data was generated from timetree.org for the 27 taxa included in the FE and ecological

210 data comparison (Kumar et al. 2017). Branch lengths (in million years) from the phylogeny were

211 used in cophenetic correlation analyses. The phylogeny was then treated as a dendrogram with

212 topology and no branch length information and subjected to correlation analysis using Baker's

213 Gamma coefficient against the FE and ecological datasets, respectively.

It is important to note that the analyses described above make several assumptions about the nature of the data: (1) that the FE simulation outcomes are known without error, (2) that the ecological variables examined are representative of feeding ecology, and that (3) the phylogenetic topology used is accurate and without error. Extensive literature examining the issues behind each of these assumptions demonstrates the complexity of each of these issues in comparative analysis (e.g., Strait et al. 2005; Heath et al. 2008; Jones et al. 2009), but for the sake of the performance-ecology trait comparison focus of this study, those factors were held constant. Furthermore, in addition to the two biomechanical outputs (ME and SE) analyzed in this study, FE simulations produce a plethora of numerical data that can be used to characterize different aspects of structural performance such as stress and strain distributions and magnitudes (Rayfield 2007; Bright 2014). A similarly broad array of ecological and life history traits are available for correlation to biomechanical performance, depending on the research question asked (Jones et al. 2009). Recognizing the diverse possibilities for applying FE analyses to study comparative biomechanics, analyses presented herein are intended to highlight the understudied issue of taxonomic sample size in comparative FE analyses using a specific case study of two skull biomechanical traits and two feeding ecological traits in carnivoran mammals. 
All data used in the analyses described above are available as supplemental files,

231

232

233

234

235

236

237

238

239

240

241

242

243

244

245

246 including the full R script for running all cluster and bootstrap analyses.

\section{Results}

ecology-based taxon clusters in the full data set is 0.0498 (bootstrapped 95\% CI: 0.0019 to

0.1260), indicating weak to no association between taxon groupings generated by FE traits versus ecological traits (Fig. 2A). Of the bootstrapped subsamples, datasets with 12 or more taxa returned correlation coefficients within the range calculated for the full dataset. Datasets with 11 taxa or fewer exhibit increasingly large correlation coefficients as sample size decreased. All taxonomic sample sizes at $\mathrm{n}=25$ or smaller contain at least 1 replicate with correlation coefficient above 0.25 , and all taxonomic sample sizes at 13 or fewer contain replicates with correlation coefficients of 0.75 or more.

Baker's Gamma correlation coefficient between FE-based clusters and phylogenetic grouping in the full dataset is 0.0171 (bootstrapped 95\% CI: 0.0033 to 0.2451494 ), indicating weak to no association between FE traits and phylogenetic structure (Fig. 2B). Resampled taxonomic datasets with 7 or more taxa returned correlation coefficient values within the range observed in the full dataset. Datasets with 6 or fewer taxa exhibited increasingly large correlation coefficients. Resampled datasets with 22 or fewer taxa contained at least one replicate with correlation coefficient above 0.25 ; datasets with 10 or fewer taxa contained at least one replicate with correlation coefficient value above 0.75 . 
252 phylogenetic grouping in the full dataset is 0.0498 (bootstrapped $95 \%$ CI: 0.0015 to 0.1037 ),

253 indicating weak to no association between ecological groupings and phylogenetic groupings

254 (Fig. 2C). Resampled datasets with 12 or more taxa returned correlation coefficient values within

255 the range observed in the full dataset. Datasets with 17 or fewer taxa contain at least one

256 replicate with correlation coefficient larger than 0.25 ; datasets with 8 taxa or fewer contain at

257 least one replicate with correlation coefficient larger than 0.75 .

258 Cophenetic correlation coefficients that consider branch length information from the

259 dendrograms returned broadly similar results to Baker's Gamma correlation coefficient analyses

260 (Fig. 2D-F). The main differences are found in (1) the FE to phylogenetic structure correlation,

261 which showed that resampled datasets with 13 taxa and above returned correlations not

262 significantly different from those estimated in the full dataset (compared to 7 taxa and above

263 using Baker's Gamma coefficient), and (2) feeding ecological to phylogenetic structure

264 correlation, which showed that resampled dataset with 10 taxa and above returned correlations

265 similar to those in the full dataset (compared to 12 taxa and above using Baker's Gamma

266 coefficient).

268 Discussion

The use of small $(<10$ taxa) comparative FE model datasets in the majority of published

270 3D FEA studies of vertebrate skulls likely results in overestimates of the feeding ecological

271 association of simulated biomechanical traits. Relative to a weak or no correlation "full" dataset

272 of 27 taxa, resampled datasets containing 11 or fewer taxa exhibit significantly elevated 
273 correlation coefficient estimates for the relationship between FE value and ecological groupings

274 (Fig. 2). Small taxonomic datasets also exhibit stronger association with phylogenetic groupings

275 in both ecological and FE-based clusters, albeit to different extents. Ecological groupings up to

$276 \sim 10$-taxon datasets show significantly higher correlation to phylogenetic groupings, and FE-

277 value based groupings show significantly higher correlation to phylogenetic groupings at

278 between 5 to 12 taxa or fewer (Fig. 2). Therefore, at small taxonomic sample sizes of 3-5 taxa,

279 there is relatively high association to phylogenetic structure in the FE data groupings compared

280 to larger taxonomic samples.

281

The elevated correlation coefficients in smaller taxonomic samples are in part driven by

282 the prevalence of outliers in the bootstrap replicates. The smaller the taxonomic sample, the

283 higher the quantity of high correlation coefficient replicates. This observation suggests taxon

284 sampling choice could have a substantial effect on the resulting presence/absence of significant

285 FE data to ecological grouping correlations. At the low end of the sampling spectrum, all three-

286 sample datasets are expected to return correlation coefficients of 0.5 or higher, with a coefficient

287 of 1.0 defining the upper quartile. This suggests that the practice of using a small number of taxa

288 to perform FE simulations to interpret the overall performance-ecology association of a larger

289 taxonomic clade runs the risk of finding spurious high correlation results when the underlying

290 full dataset exhibits weak or no correlation. In other words, the high number of outliers and the

291 elevated mean correlation coefficients at taxonomic sample sizes smaller than $\sim 11-12$ taxa

292 translate to higher incidences of false positives relative to the full dataset.

Despite the inability for small taxonomic datasets to replicate performance-ecology correlations and phylogenetic structure of larger datasets, these findings do not render small 
296 'calibrating' the research question at hand to the appropriate taxonomic sample to lessen

297 potential biases from false positives. Results from small taxonomic sample FE studies could

298 remain useful if the research question is focused on taxon-specific performance-ecology

299 comparisons, rather than extrapolation to broader clade level correlations. Nevertheless, the

300 simultaneous elevated correlation in performance-ecology, performance-phylogeny, and

301 ecology-phylogeny relationships invites caution in interpreting functional correlation when a

302 phylogenetic one is equally plausible based on available data.

To further assess whether the significant correlation of FE and feeding ecological trait

data with phylogenetic structure represents elevated phylogenetic signal at smaller taxonomic

samples, I conducted post-hoc analyses to estimate phylogenetic signal in the FE and feeding

ecology data. I used a multivariate implementation of Blomberg's K (Adams 2014) to calculate

phylogenetic signal in FE values in the full dataset as well as a similar bootstrap series of smaller

resampled datasets (Fig. 3A). Results indicate that there is a concomitant increase in $\mathrm{K}$ with

decreasing taxonomic sample size, as has been previously observed for simulated datasets

(Münkemüller et al. 2012). Increasing K and increasing FE data-feeding ecology correlation with

smaller taxon samples suggest the presence of confounding ecological and phylogenetic factors. taxon sample sizes, no elevations in phylogenetic signal are observed in either diet breadth or trophic level categorical data (Fig. 3B-C). However, the delta statistic is known to exhibit low sensitivity in detecting phylogenetic signal at small taxon sample sizes $(<20)$ (Borges et al. 2019), so it is unclear whether the feeding ecology-phylogenetic structure correlation (Fig. 2C,

$3182 \mathrm{~F}$ ) is unrelated to phylogenetic signal or whether phylogenetic signal is undetected by current 
319 methods. Comparative methods have been shown to be affected by high variance and low power

320 in estimating phylogenetic signal and other parameters at small taxon samples (Boettiger et al.

321 2012); the elevated FE data, ecology, and phylogeny correlations in the bootstrapped samples of

322 the current study (Fig. 2) appear to be similarly affected by uncertainties at small taxon sample

323 sizes.

324 Although the bootstrap analysis involves random resampling and it is not possible to

325 pinpoint which specific taxa or phylogenetic factors produce outsized effects on FE-ecology

326 correlations at small taxonomic sample sizes, some general recommendations for small-sample

327 comparative FE studies can still be made. The categorical groupings in the ecological trait data

328 were converted into a continuous distance framework in the cluster analyses via Gower distance

329 measures (see methods section). The result is a relatively clumped dendrogram with polytomies

330 representing ecologically 'identical' taxa in the context of the input variables (diet breadth and

331 trophic level). The overall dataset $(n=27)$ contains multiple samples in each ecological grouping

332 or clump, providing more evenly represented samples in each group than is possible in smaller

333 randomized resampled datasets. As such, a recommended sampling strategy for smaller

334 taxonomic datasets might be to focus on maximizing the even sampling of taxa representing

335 unique ecological trait combinations and to avoid asymmetric sampling of some ecologically

336 similar taxa over others. Such a sampling strategy should reduce spurious high-correlation

337 outcomes when using distance-based comparison methods to study FE-ecology relationships in

338 smaller datasets.

For comparative analyses using FE data to study broader clade-level associations

340 between biomechanical and ecological traits, the time-consuming nature of constructing 3D FE

341 models remains a bottleneck to achieve comparable scope to other data sources such as sequence 
342 or geometric morphometric shape data. Given the sensitivity of the correlation coefficient

343 between FE values and ecological categories on choice in taxonomic sampling and sample sizes,

344 I suggest some rethinking in future comparative FE-based biomechanics research design. Rather

345 than focusing on collection of comparative FE data in increasingly large samples of taxa, I posit

346 that most comparative biomechanics research using the FE method would be better served with a

347 theoretical morphology approach (Polly et al. 2016).

The fusion of morphospace analysis (using methods such as morphometric

morphometrics or other multivariate trait data) and FE analysis has already been demonstrated to

be a fruitful approach to test hypotheses about evolutionary optimality and the relative

importance of multiple selective forces in explaining morphological disparity (Stayton 2009;

Tseng 2013; Dumont et al. 2014; Polly et al. 2016). Theoretical morphological models

constructed at extremes and/or regular intervals over a given morphospace reduces the subjective

nature of taxon selection during FE analyses by summarizing the range of morphological

variation that underlies the subsequent FE simulation outcomes, rather than relying on

taxonomic-specific interpretations that may be more sensitive to outlier and sampling effects. In

this morphospace-driven context, the biomechanical performance context of the taxonomic dataset at hand is only indirectly dependent on the choice of taxon sampling, assuming morphospace sampling is representative of the morphological disparity in the clade studied. A shift to comparative FE analyses based on a morphospace framework leverages the comparative power of the method, especially when the practice of experimental model validation has yet to become standard practice to permit the evaluation of absolute magnitudes of FE simulation outcomes (e.g., Strait et al. 2005; Bright and Rayfield 2011). 
365

366

367

368

369

370

371

372

373

374

375

376

377

378

379

380

381

382

383

384

385

386

\section{Conclusion}

Time as a limiting factor in applications of 3D FE simulations in comparative

biomechanics research has a direct effect on limiting the taxonomic breadth of biomechanics research using comparative FE analysis. A consequence of this limitation is the presence of significant biases in performance-ecology correlation coefficients driven by small taxonomic sample sizes and outliers. Future advances in comparative biomechanics research using FE modeling may depend on a bifurcation in the application of this method. On the one hand, small taxonomic sample studies can remain useful for interpreting taxon-specific biomechanical adaptations, if carefully designed with consideration of phylogenetic structure, ecological trait representation among taxa, and preferably integrated with model validation. On the other hand, research efforts in quantifying clade-level form-function associations could be better served through theoretical morphological approaches of representing morphological disparity, rather than building increasingly large FE datasets of taxon-specific values that are more vulnerable to sampling outlier effects even at larger sample sizes. Continued improvements in model construction efficiency and accuracy are key to solving the time bottleneck issue in using FE methods in broad comparative studies. Finite element analysis is a once chic biomechanical modeling method in comparative biology that has come of age, and continued methodological and application development should help to build its analytical rigor to be on par with any other comparative methodology.

\section{Acknowledgments}


387 I thank J. Liu (UC Berkeley) and members of the Functional Anatomy and Vertebrate 388 Evolution (FAVE) Laboratory for insightful discussions on the ideas and analyses included in 389 this manuscript. J. Luo and J. Marston assisted with model construction. E. Dumont, two 390 anonymous reviewers, and editor J. Hutchinson provided wide-ranging constructive comments 391 that greatly improved all aspects of the study. All errors and insufficiency remain my own. The 392 research was funded in part by NSF DEB-1257572. 
394

395

396

397

398

399

400

401

402

403

404

405

406

407

408

409

410

411

412

413

414

\section{Literature Cited}

Adams, D. C. 2014. A Generalized K Statistic for Estimating Phylogenetic Signal from Shape and Other High-Dimensional Multivariate Data. Syst. Biol. 63:685-697.

Baker, F. B. 1974. Stability of Two Hierarchical Grouping Techniques Case 1: Sensitivity to Data Errors. J. Am. Stat. Assoc. 69:440-445. [American Statistical Association, Taylor \& Francis, Ltd.].

Boettiger, C., G. Coop, and P. Ralph. 2012. Is your phylogeny informative? Measuring the power of comparative methods. Evolution (N. Y). 66:2240-2251. John Wiley \& Sons, Ltd.

Borges, R., J. P. Machado, C. Gomes, A. P. Rocha, and A. Antunes. 2019. Measuring phylogenetic signal between categorical traits and phylogenies. Bioinformatics 35:18621869.

Bright, J. A. 2014. A review of paleontological finite element models and their validity. J. Paleontol. 88:760-769. Cambridge University Press.

Bright, J. A., and E. J. Rayfield. 2011. Sensitivity and ex vivo validation of finite element models of the domestic pig cranium. J. Anat. 219:456-471.

Dumont, E. R., I. R. Grosse, and G. J. Slater. 2009. Requirements for comparing the performance of finite element models of biological structures. J. Theor. Biol. 256:96-103.

Dumont, E. R., K. Samadevam, I. Grosse, O. M. Warsi, B. Baird, and L. M. Davalos. 2014. Selection for mechanical advantage underlies multiple cranial optima in new world leaf-nosed bats. Evolution (N. Y). 68:1436-1449.

Gower, J. C. 1971. A General Coefficient of Similarity and Some of Its Properties. Biometrics 
416

417

418

419

420

421

422

423

424

425

426

427

428

429

430

431

432

433

434

435

Grosse, I. R., E. R. Dumont, C. Coletta, and A. Tolleson. 2007. Techniques for Modeling Muscle-induced Forces in Finite Element Models of Skeletal Structures. Anat. Rec. 290:1069-1088. John Wiley \& Sons, Ltd.

Heath, T. A., S. M. Hedtke, and D. M. Hillis. 2008. Taxon sampling and the accuracy of phylogenetic analyses. J. Syst. Evol. 46:239-257.

Jones, K. E., J. Bielby, M. Cardillo, S. A. Fritz, J. O’Dell, C. D. L. Orme, K. Safi, W. Sechrest, E. H. Boakes, and C. Carbone. 2009. PanTHERIA: a species-level database of life history, ecology, and geography of extant and recently extinct mammals: Ecological Archives E090-184. Ecology 90:2648.

Kumar, S., G. Stecher, M. Suleski, and S. B. Hedges. 2017. TimeTree: A Resource for Timelines, Timetrees, and Divergence Times. Mol. Biol. Evol. 34:1812-1819.

Lauder, G. V. 1981. Form and function: structural analysis in evolutionary morphology. Paleobiology 430-442.

Lauder, G. V, and J. J. Thomason. 1995. On the inference of function from structure. Funct. Morphol. Vertebr. Paleontol. 1-18.

Morales-García, N. M., T. D. Burgess, J. J. Hill, P. G. Gill, and E. J. Rayfield. 2019. The use of extruded finite-element models as a novel alternative to tomography-based models: a case study using early mammal jaws. J. R. Soc. Interface 16:20190674.

Münkemüller, T., S. Lavergne, B. Bzeznik, S. Dray, T. Jombart, K. Schiffers, and W. Thuiller. 2012. How to measure and test phylogenetic signal. Methods Ecol. Evol. 3:743-756. John 
437

438

439

440

441

442

443

444

445

446

447

448

449

450

451

452

453

454

455

456

Pérez-Ramos, A., Z. J. Tseng, A. Grandal-D’Anglade, G. Rabeder, F. J. Pastor, and B.

Figueirido. 2020. Biomechanical simulations reveal a trade-off between adaptation to glacial climate and dietary niche versatility in European cave bears. Sci. Adv. 6:eaay9462.

Pierce, S. E., K. D. Angielczyk, and E. J. Rayfield. 2008. Patterns of morphospace occupation and mechanical performance in extant crocodilian skulls: a combined geometric morphometric and finite element modeling approach. J. Morphol. 269:840-864.

Polly, P. D., C. T. Stayton, E. R. Dumont, S. E. Pierce, E. J. Rayfield, and K. D. Angielczyk. 2016. Combining geometric morphometrics and finite element analysis with evolutionary modeling: towards a synthesis. J. Vertebr. Paleontol. 36:e1111225.

Prybyla, A. N., Z. J. Tseng, and J. J. Flynn. 2018. Biomechanical simulations of Leptarctus primus (Leptarctinae, Carnivora), and new evidence for a badger-like feeding capability. J. Vertebr. Paleontol. 38:e1531290.

Rayfield, E. J. 2007. Finite element analysis and understanding the biomechanics and evolution of living and fossil organisms. Annu. Rev. Earth Planet. Sci. 35:541-576.

Richmond, B. G., B. W. Wright, I. Grosse, P. C. Dechow, C. F. Ross, M. A. Spencer, and D. S. Strait. 2005. Finite element analysis in functional morphology. Anat. Rec. Part A Discov. Mol. Cell. Evol. Biol. An Off. Publ. Am. Assoc. Anat. 283:259-274.

Ross, C. F. 2005. Finite element analysis in vertebrate biomechanics. Anat. Rec. Part A Discov. Mol. Cell. Evol. Biol. An Off. Publ. Am. Assoc. Anat. 283:253-258.

Sokal, R. R., and F. J. Rohlf. 1962. The comparison of dendrograms by objective methods. 
Taxon 11:33-40. John Wiley \& Sons, Ltd.

458

459

460

461

462

463

464

465

466

467

468

469

470

471

472

473

474

475

476

477

Stayton, C. T. 2009. Application of thin-plate spline transformations to finite element models, or, how to turn a bog turtle into a spotted turtle to analyze both. Evol. Int. J. Org. Evol. $63: 1348-1355$.

Strait, D. S., Q. Wang, P. C. Dechow, C. F. Ross, B. G. Richmond, M. A. Spencer, and B. A. Patel. 2005. Modeling elastic properties in finite-element analysis: how much precision is needed to produce an accurate model? Anat. Rec. Part A Discov. Mol. Cell. Evol. Biol. An Off. Publ. Am. Assoc. Anat. 283:275-287.

Thomason, J. J. 1991. Cranial strength in relation to estimated biting forces in some mammals. Can. J. Zool. 69:2326-2333. NRC Research Press.

Tseng, Z. J. 2013. Testing adaptive hypotheses of convergence with functional landscapes: a case study of bone-cracking hypercarnivores. PLoS One 8:e65305.

Tseng, Z. J., and J. J. Flynn. 2015. Are Cranial Biomechanical Simulation Data Linked to Known Diets in Extant Taxa? A Method for Applying Diet-Biomechanics Linkage Models to Infer Feeding Capability of Extinct Species. PLoS One 10:e0124020. Public Library of Science.

Tseng, Z. J., D. F. Su, X. Wang, S. C. White, and X. Ji. 2017. Feeding capability in the extinct giant Siamogale melilutra and comparative mandibular biomechanics of living Lutrinae. Sci. Rep. 7:15225.

Wroe, S., C. McHenry, and J. Thomason. 2005. Bite club: comparative bite force in big biting mammals and the prediction of predatory behaviour in fossil taxa. Proc. R. Soc. B Biol. Sci. 
479

480 
481 TABLE 1. Biomechanical attributes from finite element simulations used in bootstrap analyses.

482 ACME, adjusted canine mechanical efficiency; AP4ME, adjusted premolar four mechanical

483 efficiency; ADJCSE, adjusted canine strain energy (in Joules); ADJP4SE, adjusted premolar four

484 strain energy (in Joules).

\begin{tabular}{|c|c|c|c|c|c|c|}
\hline Genus & Species & ACME & AP4ME & ADJCSE & ADJP4SE & Reference \\
\hline Ailuropoda & melanoleuca & 0.1688 & 0.2453 & 0.5243 & 0.4685 & Perez Ramos et al., 2020 \\
\hline Ailurus & fulgens & 0.1664 & 0.2588 & 0.5762 & 0.5442 & Prybyla et al., 2018 \\
\hline Aonyx & capensis & 0.2483 & 0.3566 & 0.9297 & 1.1842 & This study \\
\hline Bassariscus & astutus & 0.1458 & 0.2380 & 0.4134 & 0.4425 & Prybyla et al., 2018 \\
\hline Canis & lupus & 0.1032 & 0.1834 & 0.8375 & 0.7801 & Prybyla et al., 2018 \\
\hline Canis & mesomelas & 0.1507 & 0.2454 & 1.1223 & 0.9307 & Prybyla et al., 2018 \\
\hline Crocuta & crocuta & 0.1759 & 0.2894 & 0.4261 & 0.4750 & Prybyla et al., 2018 \\
\hline Gulo & gulo & 0.2585 & 0.3552 & 0.3664 & 0.3003 & Prybyla et al., 2018 \\
\hline Helarctos & malayanus & 0.1678 & 0.2253 & 0.5731 & 0.4959 & Perez Ramos et al., 2020 \\
\hline Herpestes & javanicus & 0.1186 & 0.1885 & 0.6201 & 0.5672 & Prybyla et al., 2018 \\
\hline Hydrictis & maculicollis & 0.3042 & 0.5123 & 1.1761 & 2.4438 & This study \\
\hline Lutra & lutra & 0.2000 & 0.3122 & 1.1488 & 1.1752 & This study \\
\hline Lycaon & pictus & 0.2056 & 0.3179 & 1.2854 & 1.2611 & Prybyla et al., 2018 \\
\hline Melursus & ursinus & 0.1706 & 0.2412 & 0.6626 & 0.6374 & Perez Ramos et al., 2020 \\
\hline Mephitis & mephitis & 0.1032 & 0.1397 & 0.8477 & 0.8436 & Prybyla et al., 2018 \\
\hline Panthera & pardus & 0.0850 & 0.1430 & 0.5437 & 0.4759 & Prybyla et al., 2018 \\
\hline Parahyaena & brunnea & 0.1724 & 0.3306 & 0.5206 & 0.6353 & Prybyla et al., 2018 \\
\hline Potos & flavus & 0.2637 & 0.3716 & 1.8570 & 1.2252 & Prybyla et al., 2018 \\
\hline Procyon & lotor & 0.1162 & 0.1634 & 0.8562 & 0.7668 & Prybyla et al., 2018 \\
\hline Spilogale & putorius & 0.1059 & 0.1491 & 0.2593 & 0.2534 & Prybyla et al., 2018 \\
\hline Taxidea & taxus & 0.3404 & 0.5395 & 0.5622 & 0.4336 & Prybyla et al., 2018 \\
\hline Tremarctos & ornatus & 0.1423 & 0.1914 & 0.4451 & 0.4271 & Perez Ramos et al., 2020 \\
\hline Urocyon & cinereoargenteus & 0.1395 & 0.2243 & 1.0640 & 0.9317 & Prybyla et al., 2018 \\
\hline Ursus & americanus & 0.1422 & 0.2015 & 0.5321 & 0.6297 & Perez Ramos et al., 2020 \\
\hline Ursus & arctos & 0.1647 & 0.1824 & 0.5123 & 0.4940 & Perez Ramos et al., 2020 \\
\hline Ursus & maritimus & 0.1248 & 0.1868 & 0.4922 & 0.5513 & Perez Ramos et al., 2020 \\
\hline Ursus & thibetanus & 0.1429 & 0.2038 & 0.5227 & 0.4759 & Perez Ramos et al., 2020 \\
\hline
\end{tabular}

485

486 
487 TABLE 2. Feeding ecological variable definitions from the PanTHERIA database (Jones et al.

488 2009).

\section{Ecological}

Variable

"Number of dietary categories eaten by each

Diet Breadth species. Categories were defined as vertebrate, invertebrate, fruit, flowers/nectar/pollen, leaves/branches/bark, seeds, grass and roots/tubers."

"Trophic level of each species: (1) herbivore

Trophic Level (not vertebrate and/or invertebrate), (2) omnivore (vertebrate and/or invertebrate plus

1 (dietary specialist) to 6 (dietary generalist) any of the other categories) and (3) carnivore

(1) herbivore (2) omnivore (3) carnivore (vertebrate and/or invertebrate only. 
492

493

494

495

496

497

498

499

500

501

502

503

504

505

506

507

508

509

510

511

512

FIGURE 1. Histograms of publications on finite element analysis from 2005-2020. A. Total number of publications from each year in the Web of Science database (searched 22 December 2020) using the key words "finite element" + "evolution" + bio*. B. Number of taxa included in each publication on vertebrate skull biomechanics listed in Web of Science (searched 15 December 2020) using key words "finite element" + "skull" + phylo*.

FIGURE 2. Correlation coefficients calculated in bootstrap analyses of subsampled datasets in $\mathbf{A}$. FE data versus feeding ecology using Baker's Gamma, B. FE data versus phylogeny using Baker's Gamma, C. Feeding ecology versus phylogeny using Baker's Gamma, D. FE data versus feeding ecology using cophenetic correlation, E. FE data versus phylogeny using cophenetic correlation, F. Feeding ecology versus phylogeny using cophenetic correlation. Red solid line indicates correlated coefficient value in the full dataset, dotted red lines represent $95 \%$ confidence intervals. Blue bars represent $95 \%$ confidence intervals of mean correlation coefficient values at each taxonomic sample size. Boxplots show median values and interquartile ranges. Asterisks indicate sample size above which subsample and full dataset produce similar correlation coefficient values on average.

Figure 3. Phylogenetic signal in bootstrap analyses of subsampled datasets in A. FE data, B. Diet breadth data, and $\mathbf{C}$. Trophic level data. Red solid line indicates correlated coefficient value in the full dataset, dotted red lines represent $95 \%$ confidence intervals. Blue bars represent $95 \%$ confidence intervals of mean correlation coefficient values at each taxonomic sample size. Boxplots show median values and interquartile ranges. 


\section{SUPPLEMENTAL DATA FILES}

514 TABLE S1. Finite element analysis literature meta-analysis results.

515 TABLE S2. Elastic moduli sensitivity analysis results.

516 TABLE S3. Raw versus moduli standardized results.

517 FIGURE S1. Linear regression models for the relationship between finite element results with 18

518 and $20 \mathrm{GPa}$ elastic moduli values. A. Mechanical efficiency values, B. Strain energy values.

519 File: Tseng_PeerJ_FEA_R_script_revised.R

520 File: Tseng_PeerJ_FE_Dataset.csv

521 File: Tseng_PeerJ_Eco_Dataset.csv

522 File: Tseng_PeerJ_timetree.nwk

523 
Figure 1

Histograms of publications using FE analysis from 2005-2020

A. Total number of publications from each year in the Web of Science database (searched 22 December 2020) using the key words "finite element" + "evolution" + bio*. B. Number of taxa included in each publication on vertebrate skull biomechanics listed in Web of Science (searched 15 December 2020) using key words "finite element" + "skull" + phylo*. 

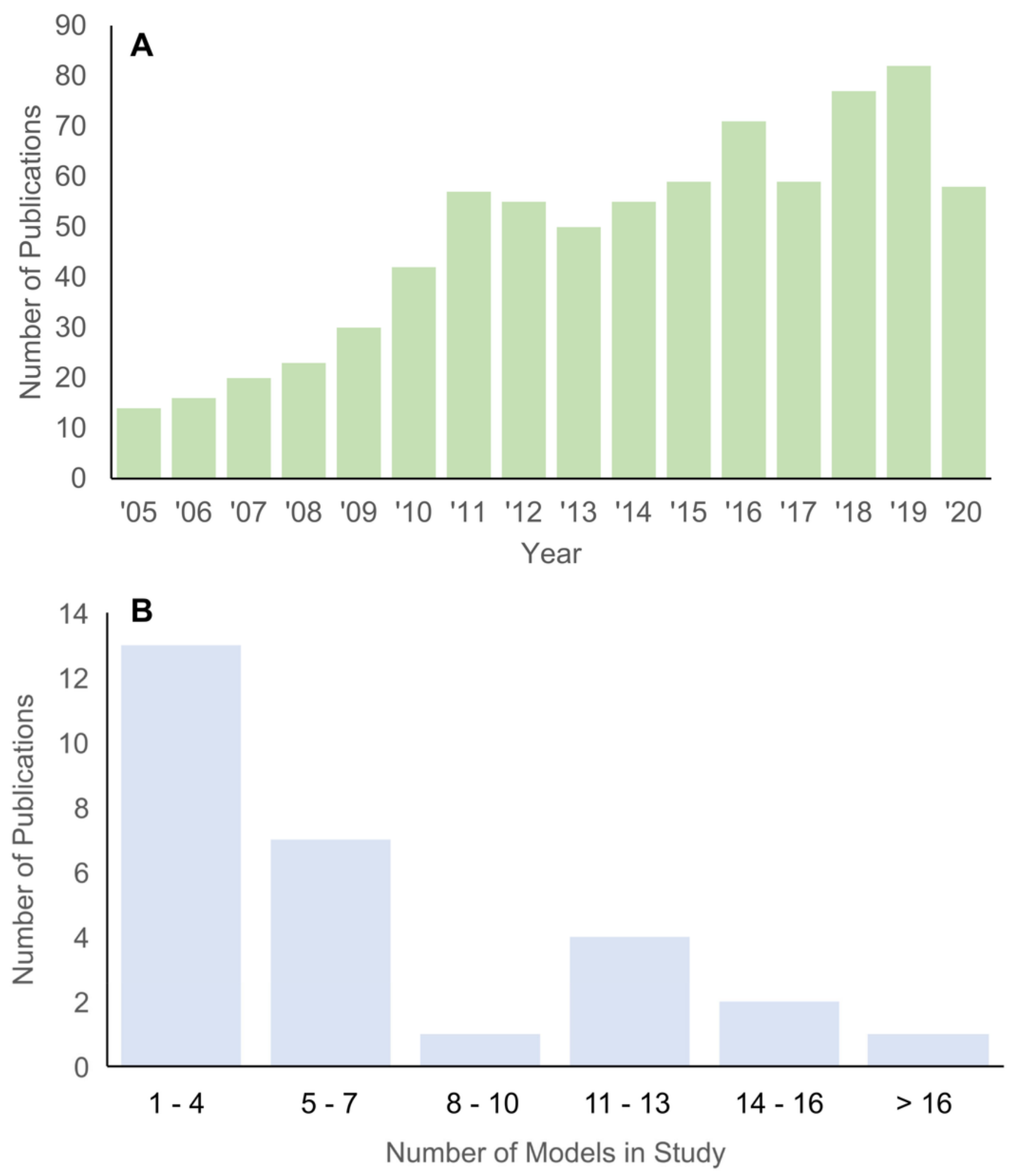


\section{Figure 2}

Correlation coefficients calculated in bootstrap analyses of subsampled datasets.

A. FE data versus feeding ecology using Baker's Gamma, B. FE data versus phylogeny using

Baker's Gamma, C. Feeding ecology versus phylogeny using Baker's Gamma, D. FE data versus feeding ecology using cophenetic correlation, E. FE data versus phylogeny using cophenetic correlation, F. Feeding ecology versus phylogeny using cophenetic correlation. Red solid line indicates correlated coefficient value in the full dataset, dotted red lines represent $95 \%$ confidence intervals. Blue bars represent $95 \%$ confidence intervals of mean correlation coefficient values at each taxonomic sample size. Boxplots show median values and interquartile ranges. Asterisks indicate sample size above which subsample and full dataset produce similar correlation coefficient values on average. 

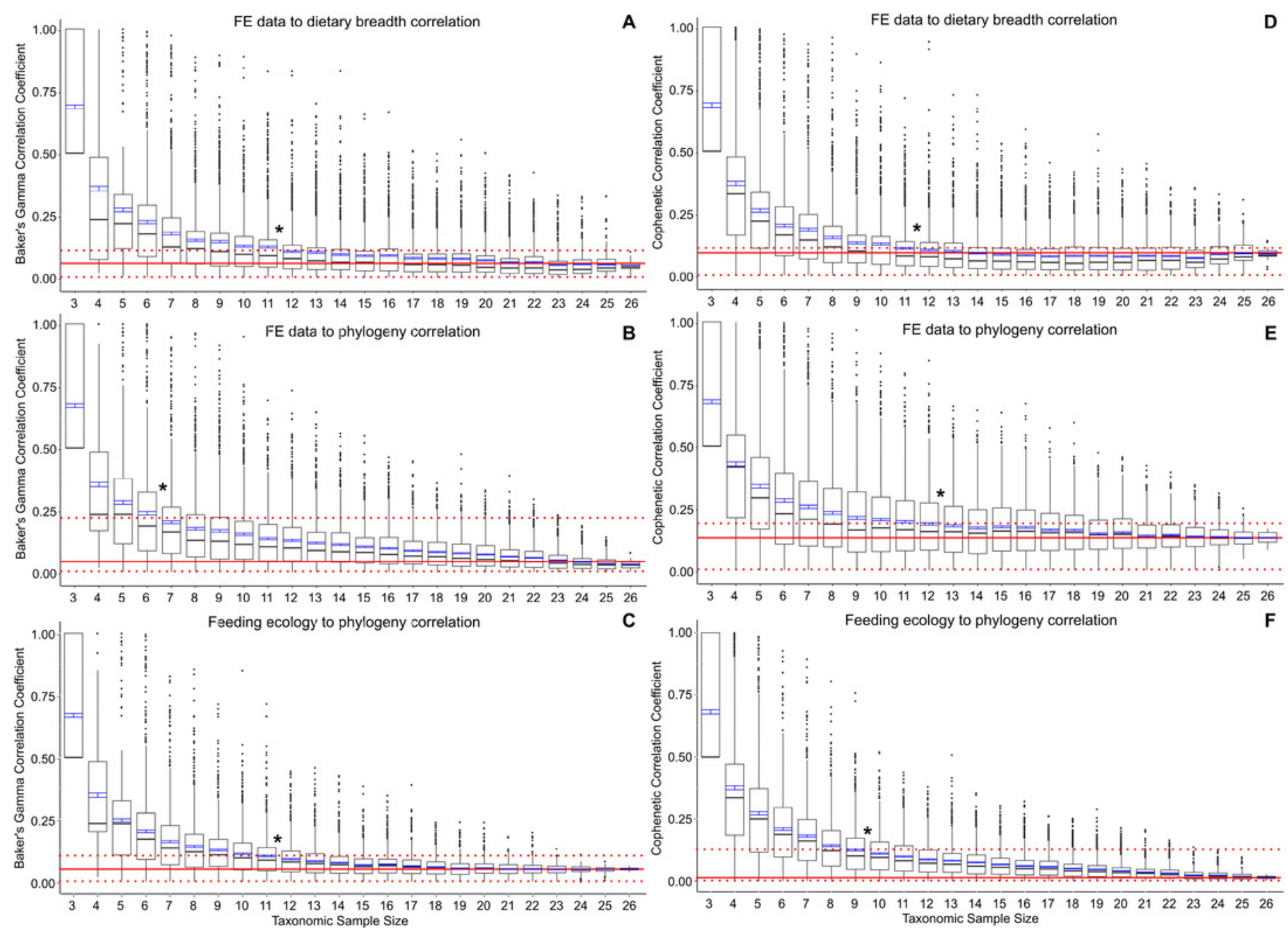


\section{Figure 3}

Phylogenetic signal in bootstrap analyses of subsampled datasets

A. FE data, B. Diet breadth data, and C. Trophic level data. Red solid line indicates correlated coefficient value in the full dataset, dotted red lines represent $95 \%$ confidence intervals. Blue bars represent $95 \%$ confidence intervals of mean correlation coefficient values at each taxonomic sample size. Boxplots show median values and interquartile ranges. 

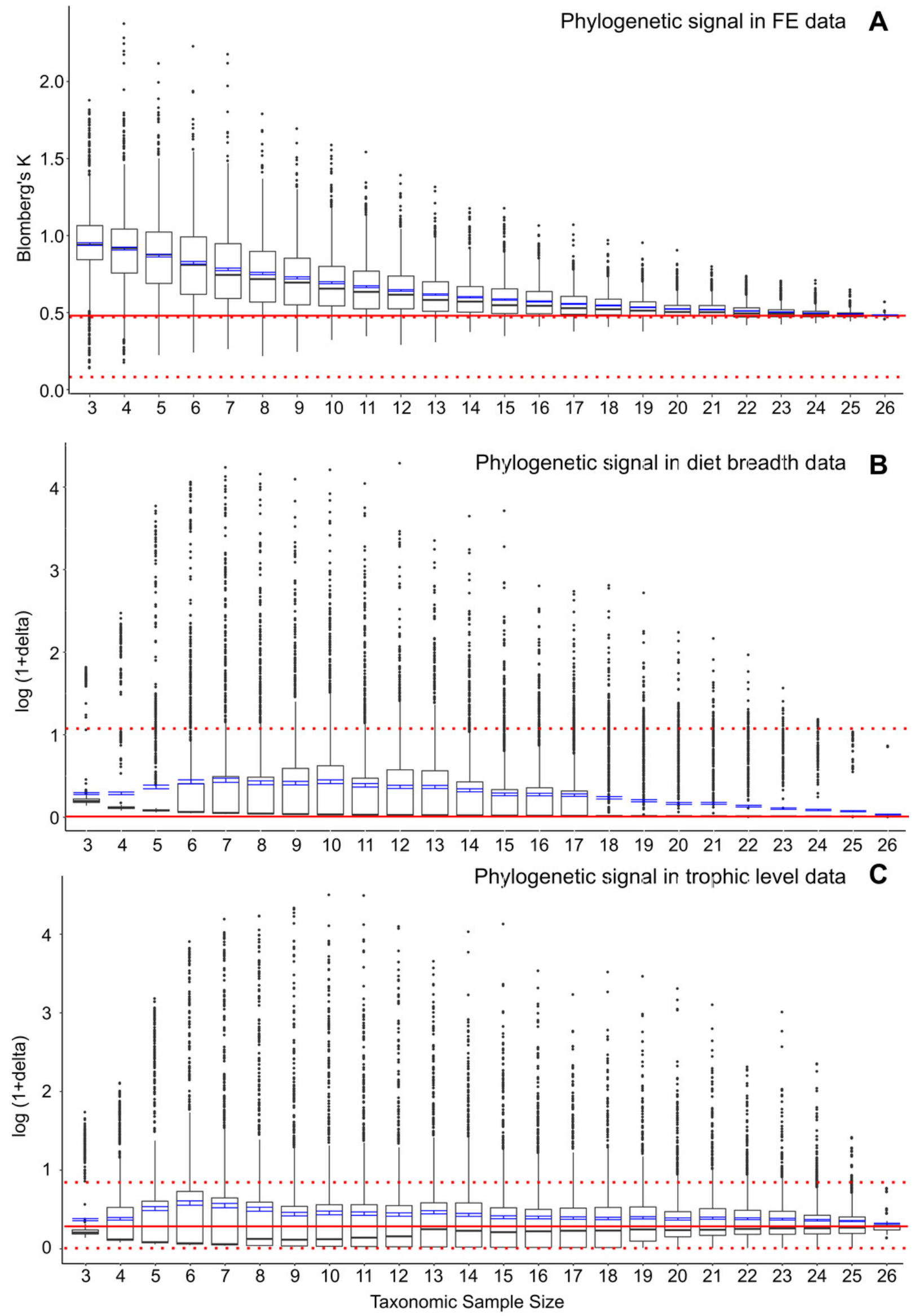


\section{Table $\mathbf{1}$ (on next page)}

Biomechanical attributes from finite element simulations used in bootstrap analyses

ACME, adjusted canine mechanical efficiency; AP4ME, adjusted premolar four mechanical efficiency; ADJCSE, adjusted canine strain energy (in Joules); ADJP4SE, adjusted premolar four strain energy (in Joules). 


\begin{tabular}{|c|c|c|c|c|c|c|}
\hline Genus & Species & ACME & AP4ME & ADJCSE & ADJP4SE & Reference \\
\hline Ailuropoda & melanoleuca & 0.1688 & 0.2453 & 0.5243 & 0.4685 & Perez Ramos et al., 2020 \\
\hline Ailurus & fulgens & 0.1664 & 0.2588 & 0.5762 & 0.5442 & Prybyla et al., 2018 \\
\hline Aonyx & capensis & 0.2483 & 0.3566 & 0.9297 & 1.1842 & This study \\
\hline Bassariscus & astutus & 0.1458 & 0.2380 & 0.4134 & 0.4425 & Prybyla et al., 2018 \\
\hline Canis & lupus & 0.1032 & 0.1834 & 0.8375 & 0.7801 & Prybyla et al., 2018 \\
\hline Canis & mesomelas & 0.1507 & 0.2454 & 1.1223 & 0.9307 & Prybyla et al., 2018 \\
\hline Crocuta & crocuta & 0.1759 & 0.2894 & 0.4261 & 0.4750 & Prybyla et al., 2018 \\
\hline Gulo & gulo & 0.2585 & 0.3552 & 0.3664 & 0.3003 & Prybyla et al., 2018 \\
\hline Helarctos & malayanus & 0.1678 & 0.2253 & 0.5731 & 0.4959 & Perez Ramos et al., 2020 \\
\hline Herpestes & javanicus & 0.1186 & 0.1885 & 0.6201 & 0.5672 & Prybyla et al., 2018 \\
\hline Hydrictis & maculicollis & 0.3042 & 0.5123 & 1.1761 & 2.4438 & This study \\
\hline Lutra & lutra & 0.2000 & 0.3122 & 1.1488 & 1.1752 & This study \\
\hline Lycaon & pictus & 0.2056 & 0.3179 & 1.2854 & 1.2611 & Prybyla et al., 2018 \\
\hline Melursus & ursinus & 0.1706 & 0.2412 & 0.6626 & 0.6374 & Perez Ramos et al., 2020 \\
\hline Mephitis & mephitis & 0.1032 & 0.1397 & 0.8477 & 0.8436 & Prybyla et al., 2018 \\
\hline Panthera & pardus & 0.0850 & 0.1430 & 0.5437 & 0.4759 & Prybyla et al., 2018 \\
\hline Parahyaena & brunnea & 0.1724 & 0.3306 & 0.5206 & 0.6353 & Prybyla et al., 2018 \\
\hline Potos & flavus & 0.2637 & 0.3716 & 1.8570 & 1.2252 & Prybyla et al., 2018 \\
\hline Procyon & lotor & 0.1162 & 0.1634 & 0.8562 & 0.7668 & Prybyla et al., 2018 \\
\hline Spilogale & putorius & 0.1059 & 0.1491 & 0.2593 & 0.2534 & Prybyla et al., 2018 \\
\hline Taxidea & taxus & 0.3404 & 0.5395 & 0.5622 & 0.4336 & Prybyla et al., 2018 \\
\hline Tremarctos & ornatus & 0.1423 & 0.1914 & 0.4451 & 0.4271 & Perez Ramos et al., 2020 \\
\hline Urocyon & cinereoargenteus & 0.1395 & 0.2243 & 1.0640 & 0.9317 & Prybyla et al., 2018 \\
\hline Ursus & americanus & 0.1422 & 0.2015 & 0.5321 & 0.6297 & Perez Ramos et al., 2020 \\
\hline Ursus & arctos & 0.1647 & 0.1824 & 0.5123 & 0.4940 & Perez Ramos et al., 2020 \\
\hline Ursus & maritimus & 0.1248 & 0.1868 & 0.4922 & 0.5513 & Perez Ramos et al., 2020 \\
\hline Ursus & thibetanus & 0.1429 & 0.2038 & 0.5227 & 0.4759 & Perez Ramos et al., 2020 \\
\hline
\end{tabular}


Table 2 (on next page)

Feeding ecological variable definitions from the PanTHERIA database (Jones et al. 2009) 


\section{Ecological}

Variable

"Number of dietary categories eaten by each

Diet Breadth species. Categories were defined as vertebrate, invertebrate, fruit, flowers/nectar/pollen, leaves/branches/bark, seeds, grass and roots/tubers."

"Trophic level of each species: (1) herbivore

Trophic Level (not vertebrate and/or invertebrate), (2) omnivore (vertebrate and/or invertebrate plus any of the other categories) and (3) carnivore (vertebrate and/or invertebrate only.
1 (dietary specialist) to 6 (dietary generalist) 